\title{
Polymeric capacitive transducers and arrays for gas coupled operation
}

\author{
Richard L O'Leary \\ Dept of Electronic and Electrical Engineering \\ University of Strathclyde, \\ Glasgow, UK
}

\author{
Gerald Harvey \\ Weidlinger Associates \\ Glasgow, UK
}

\begin{abstract}
This paper describes a facile approach for the manufacture of capacitive transducers based on micropatterning of polymer substrates. The process affords independent control of cavity depth, lateral dimensions and spatial distribution. The manufacture of air-coupled devices is guided by PZFlex modeling based on the range cavity dimension available from the method. Single element devices, operating at $500 \mathrm{kHz}$, exhibit transducer bandwidths in excess of $100 \%$ and two-way insertion loss of $62 \mathrm{~dB}$ being typical. Laser vibrometery confirms uniform surface dilation of the excited. PZFlex, configured with MPI parallelization scheme, has been used to assess linear array performance inter-element cross talk is minimal and observed to be $21 \mathrm{~dB}$.
\end{abstract}

Keywords-capacitive transducer; modelling; finite element; aircoupled ultrasound

\section{INTRODUCTION}

Capacitive ultrasonic transducers typically comprise a membrane positioned above a substrate patterned with a regular array of uniformly dimensioned cavities. The cavity lateral dimensions and depth, together with the elastic properties of the membrane will govern the vibrational behavior and dominant frequency of the transducer arrangement. Ultrasound transmission and reception is afforded via controlled electrical or mechanical stimulus of the membrane, respectively.

The literature details a range of manufacturing techniques for the creation of the cavity structure. Micro-machining of silicon [2], [3], controlled polishing [1] to create a substrate with a known surface roughness; microstereolithography (MSL) [4] or the multi-user MEMS process (mumps) [5]. The commonality of each technique is the creation of an array of cavities with uniform geometry, dimensions and spatial arrangement. Each of the reported techniques has its own relative merits and inherent limitations and there is no panacea for the facile creation of capacitive devices across a wide range of length scales. More recently, a methodology employing phase separation of ternary polymer solutions yielded limited success for substrate manufacture - the method lacked the ability to independently control the depth and lateral dimensions of the resultant cavities. This resulted in devices being limited to $\sim 200 \mathrm{kHz}[6]$.

This paper describes a novel approach to the problem via micro-patterning of polymer substrates [8] for the manufacture of capacitive devices. A piezoelectric droplet generator is employed to create a regular array of sessile droplets on a polymer substrate, the droplet act as a positive mask of the desired cavity microstructure. The substrate is then exposed to a saturated solvent vapour, adsorption of the solvent causes swelling of the polymer surface except in the areas where the droplets are situated. Controlling the time the polymer is exposed to the solvent vapour controls the depth of the resultant cavities; the droplet size controls the lateral dimension. Importantly, cavity diameter and depth can be controlled independently therefore the methodology has the potential to create a wide array of devices. In order to explore the range of potential device configurations the PZFlex finite element (FE) code has been employed using a a custom cloud computing architecture allowing for 100's of simultaneous parallel simulations, has been employed to explore the problem space [7] This study indicated that devices suited to air-coupled operation, center frequencies in the sub- $1 \mathrm{MHz}$ band, could be readily manufactured

In the next Section the manufacturing methodology will be outlined along with potential transducer operational frequency determined from the FE modeling exercise [7]. Air-coupled transducers with nominal frequencies of operation in $\sim 500 \mathrm{kHz}$ will be presented in both pulse echo and pitch catch mode. Laser vibrometery confirms the devices possess uniform surface dilation. Finally, the prospects for such device to be employed in array structures will be investigated via a new approach to FE modeling of array manufactured by example devices will be presented.

\section{METHODOLOGY}

Polystyrene, 3mm sheet (Amari Plastics, Glasgow), was cut to the desired dimensions and cleaned via sonication in methanol (Sigma Aldrich, Dorset, UK) and air dried at room temperature. Solutions of ethylene glycol (Sigma Aldrich, Dorset, UK) $40 \% \mathrm{v} / \mathrm{v}$ in water were prepared.

A piezoelectric tube actuator, (PT120, PI, Germany) fitted with a continuous glass capillary was employed as a droplet generator (DG) [9]. In order to create the nozzle of the DG, one end of the glass capillary was shaped by gently heating in a propane flame. Carefully controlling the time of heat the capillary causes the end of the shrink to form a nozzle. The nozzle is polished using $1 \mu \mathrm{m}$ alumina to create a flat surface at the outlet [10]. Figure 1. details a photomicrograph of a nozzle, 
in this example the nozzle diameter is $150 \mu \mathrm{m}$. The glass capillary was bonded into the piezoelectric actuator tube using Araldite Rapid epoxy (Huntsman, Cambridge, UK).

An Agilent 33220A function generator (Agilent, South Queensferry, UK) was used to supply a $90 \mathrm{mV} 3 \mu$ s pulse to an ENI power amplifier (ENI details), $-300 \mathrm{~V}$ impulse being supplied to the DG. A single pulse resulted in droplet of $120 \mu \mathrm{m}$ being deposited onto the polystyrene substrate as shown in Figure 2. Larger droplet diameters were achieved by depositing multiple droplets in the same location. This approach allows control of the diameter of individual cavities in the polymer substrate. The diameter of the deposited droplet does not increase monotonically with the number of droplets; this is a result of the wetting of the droplet to the polystyrene surface.

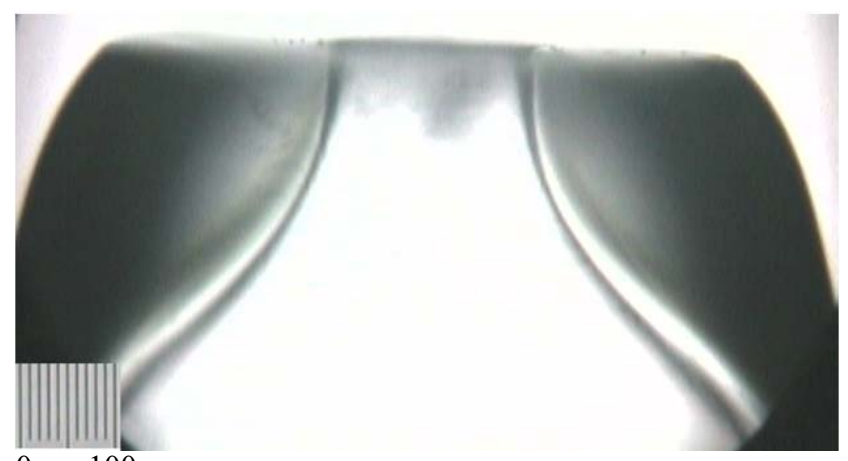

$0 \quad 100 \mu \mathrm{m}$

Figure 1. Photomicrograph of the tip of the glass capillary

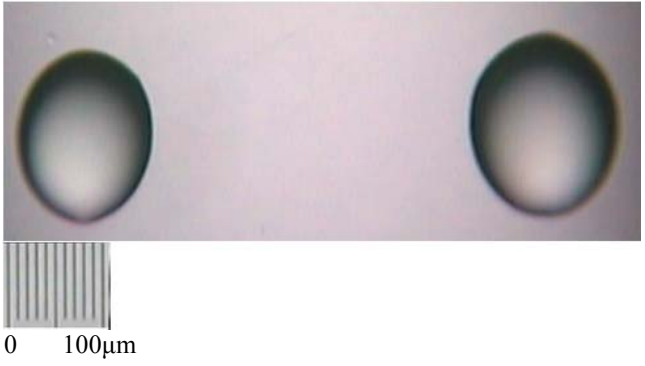

Figure 2. Photomicrograph of a droplet of ethylene glycol solution despsited onto polystyrene substrate

In order to control the spatial distribution of sessile droplets, and hence cavities, the DG was mounted in a Colinbus profiler (Colinbus, Belgium) a graphical user interface (GUI) was created using the LabVIEW environment (National Instruments). Communication to the Colinbus profiler was undertaken using the RS-232 protocol to control of the position and pitch. The LabVIEW GUI interfaced to the Agilent 33220 via USB in order to excite the DG in the appropriate manner to control the dimensions of the deposited droplets.

Once the droplets of ethylene glycol solution had been deposited onto the polystyrene the sample was exposed to a vapor of toluene, during this process the polystyrene substrate absorbs toluene causing it to swell. The regions of polystyrene under the deposited droplets do not absorb toluene and hence the do not swell thus forming small cavities within the surface of the polystyrene sample. After exposure to toluene vapor, the sample is air dried at $20^{\circ} \mathrm{C}$ and then washed by sonication in deionized water and further air-drying at $20^{\circ} \mathrm{C}$. As will be shown in the next section, the period of time the sample is exposed to the toluene vapor governs the extent of the swelling and hence depth of the cavity.

\section{CAVITY CHARACTERISATION}

The cavities were characterized using a Veeco NT1100 optical profiler. This instrument provides a rapid, non-contact method of measuring the height profile of an area (up to a few $\mathrm{mm} 2$ ) using white light interferometry with a resolution in the vertical axis of $0.1 \mathrm{~nm}$. Figure 3 . illustrates the variation in cavity depth as a function of toluene exposure. The data show a non-linear variation as a function of exposure time. However, the depth of the cavities observed here differ somewhat from those observed by elsewhere [8], this may be a result of the specific grade of polystyrene employed in this study. As expected, cavity depth was found to be independent of cavity diameter. In addition to depth, the surface roughness of the samples was also assessed. The RMS surface roughness of the polystyrene surface that was exposed to toluene vapor was found to be $10 \mathrm{~nm}$ irrespective of the exposure time. The RMS surface roughness of the floors of the resultant cavities was also found to be $10 \mathrm{~nm}$.

It is clear that the methodology is capable to manufacturing substrates with a wide range of cavity dimensions, suited to the manufacture of a range of dimensions achievable using the described methodology was used employed in a PZFlex model to explore the potential for the use of the micro-patterned substrates in the manufacture of capacitive ultrasonic transducers.

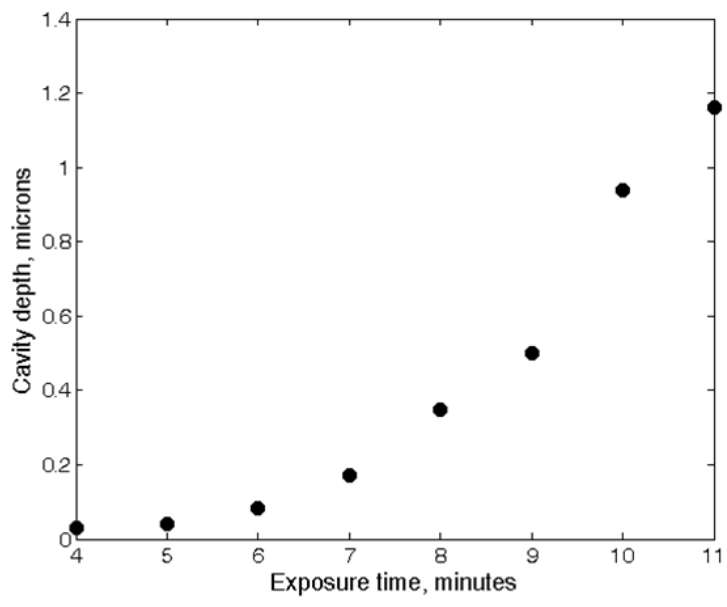

Figure 3. Cavity depth as a function of exposure time

\section{AIR-COUPLED TRANSDUCERS}

PZFlex modeling was used as a guide to the manufacture of capacitive devices operating in the $500 \mathrm{kHz}$ frequency range for air-coupled inspection. Example devices were manufactured; Table 1 details the constructional parameters of 
two example devices that will be described. In each of the example devices, the Colinbus profiler equipped with the DG was used to deposit uniform droplets onto $30 \mathrm{~mm}$ diameter sample of polystyrene. Once patterned, the polystyrene sample was exposed to toluene vapor to create the desired cavity depth in line with the data in Figure 3. After the solvent exposure, washing and drying, the polystyrene substrate is then electroded on all faces with silver using an electroless process with a tin sensitizer [11]. The micro-patterned polystyrene was then fitted with 8 micron Kapton membrane (Goodfellow, Huntingdon, UK) the outer face of the dielectric membrane has a sputtered aluminum electrode. Figure 5. shows a photograph of a completed device.
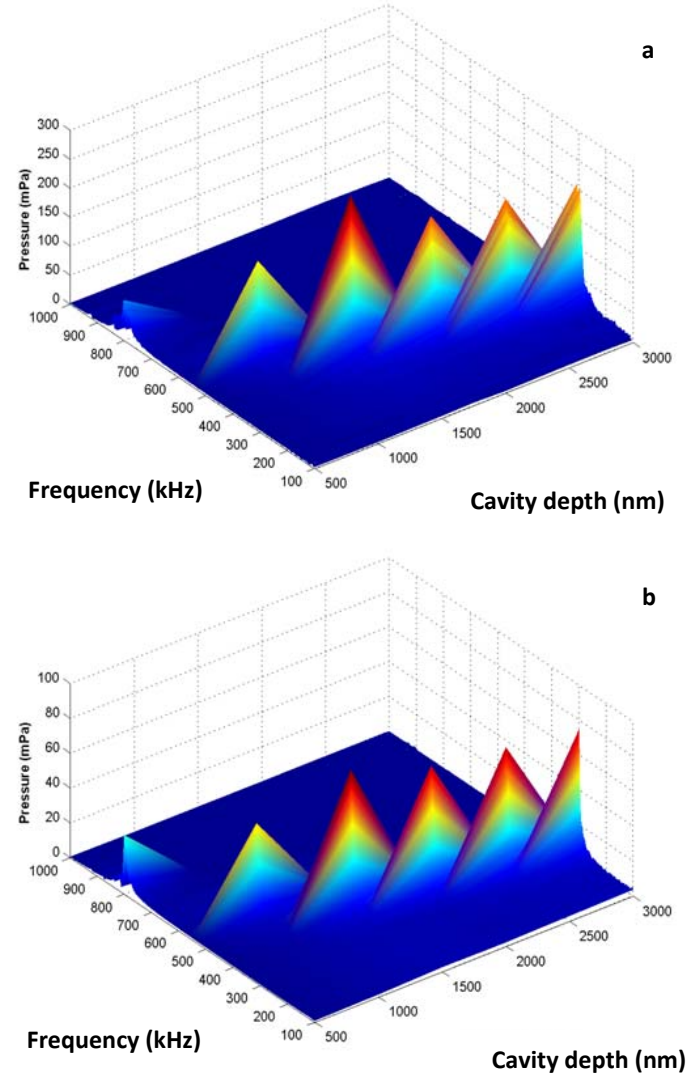

Figure 4. FE derived frequency response for a range of cavity depths for: (a) 1000 micron diameter cavities and (b) 500micron diameter cavities

TABLE I. CONSTRUCTIONAL PARAMETERS OF EXAMPLE DECVIES

\begin{tabular}{|l|c|c|}
\hline & Device A & B \\
\hline $\begin{array}{l}\text { Cavity diameter, } \\
\text { microns }\end{array}$ & 500 & 1000 \\
\hline $\begin{array}{l}\text { Target cavity depth, } \\
\text { microns }\end{array}$ & 0.7 & 0.9 \\
\hline $\begin{array}{l}\text { Actual cavity depth, } \\
\text { microns }\end{array}$ & 0.7 & 1800 \\
\hline Cavity pitch, microns & 1000 & 1 \\
\hline
\end{tabular}

The performance of the two devices was assessed using air coupled pulse echo response from a $50 \mathrm{~mm}$ glass block. In each case a $200 \mathrm{~V}$ bias plied from a Brandenburg $475 \mathrm{R}$ with a Panametrics pulser receiver model 5052PR being used to excite the membrane. The time and frequency domain data for devices A and B are shown in Figure 6.

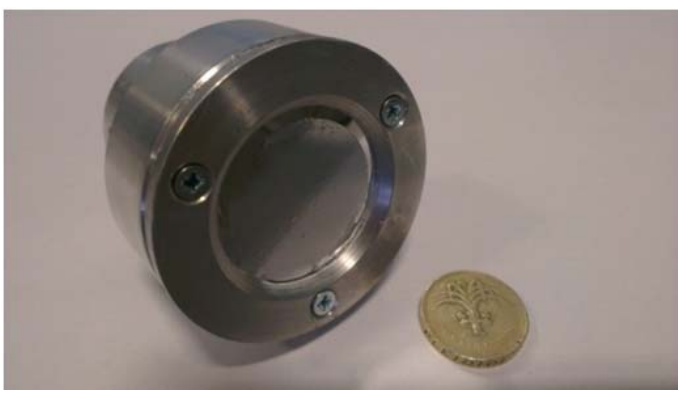

Figure 5. Photgraph of a completed device
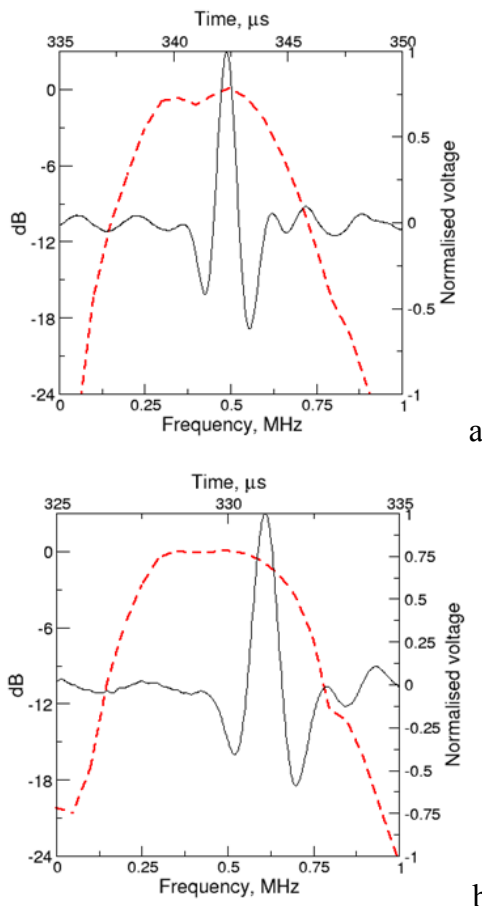

Figure 6. Experimental air-coupled impulse response data: (a) device A (b) device B

It is clear that both devices are capable of generating ultrasound in the desired frequency range. In order to further investigate their potential a second device of design $\mathrm{A}$, detailed in Table 1, was manufactured to assess the pitch-catch performance, with the results for impulse excitation being detailed in Figure 7. (a). To assess the insertion loss of the pair of devices, a $10 \mathrm{~V}_{\mathrm{pp}}, 2$-cycle tone burst at $450 \mathrm{kHz}$ was used to excite the transmitter, the data acquired at the receiver after amplification via a Panametrics 5052PR are presented in Figure 7. (b). Receive signal was found to be $794 \mathrm{mV}_{\mathrm{pp}}$ giving an insertion loss of $-62 \mathrm{~dB}$. Finally laser vibrometry was performed using an OFV 056 (Polytec, Germany) to determine the surface dilation of the membrane, the magnitude and phase of the surface dilation for device A is shown in Figure 8. The 
peaks in the magnitude data correspond to each individual cavity - importantly the phase response is uniform.
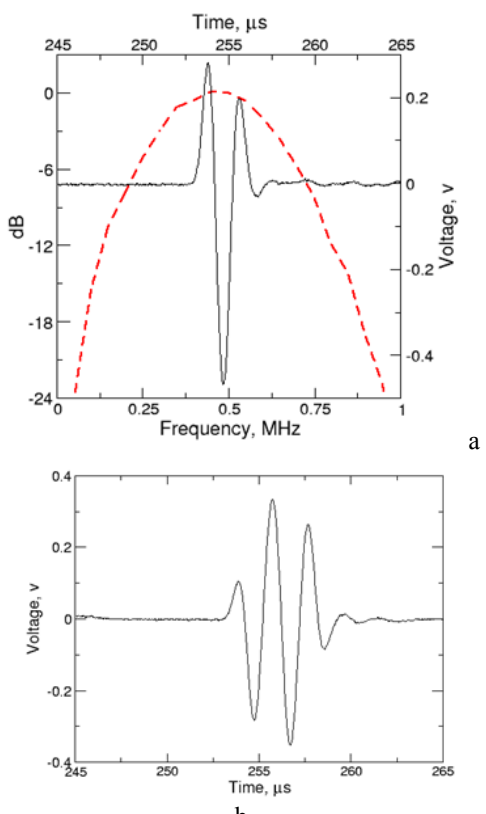

b

Figure 7. Experimental pitch catch rsponse for a pair of devices of design A, (a) impulse excitation (b) toneburst excitation $10 \mathrm{~V}_{\mathrm{pp}}, 2$-cycle at $450 \mathrm{kHz}$

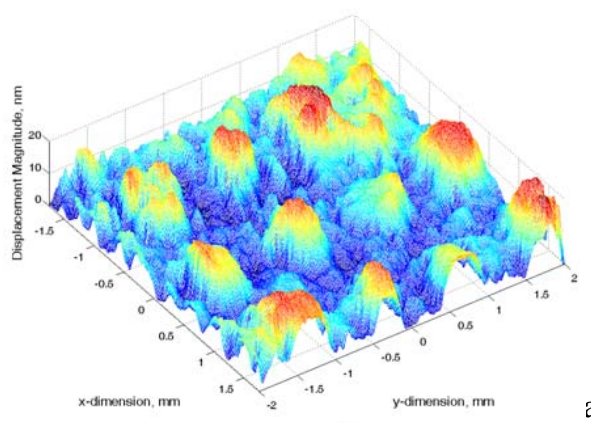

a

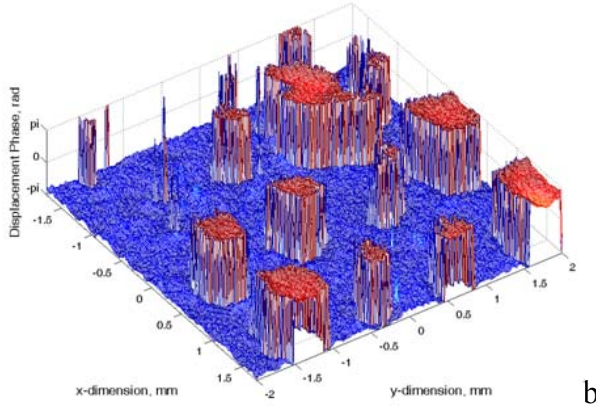

Figure 8. Surface dilation of the membrane for device A: (a) displacement magnitude (b) phase

\section{ARRAY MODELLING}

The PZFlex modeling suite was employed to construct a 3D model of a linear array of cavities in order to understand the extent of inter-element cross talk in these devices when configured as an array. In the each element in the array was implemented using MPI protocol in order to parallelize the calculations and reduce the time to solution. In the simulation one element in the array was excited and the membrane displacement monitored on the excited element and adjacent unexcited elements. Figure 9. details the results of the FE model, comparing the surface dilation data indicates that the cross talk is $22 \mathrm{~dB}$ for this configuration.

\section{CONCLUDING REMARKS}

This paper has presented a facile approach for the manufacture of capacitive transducers employing a sessile droplet polymer mask deposited upon a polymer substrate. Capacitive devices with a nominal center frequency of $500 \mathrm{kHz}$ exhibit insertion loss of $62 \mathrm{~dB}$ and uniform surface dilation - important for beam characteristics. FE modeling has been employed to assess array crosstalk and this is predicted to be $22 \mathrm{~dB}$.
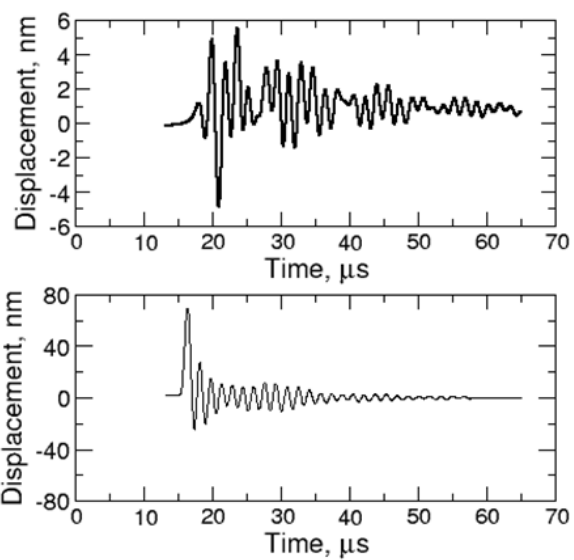

Figure 9. PZFlex derived surface dilation of the membrane for an array based on device A: lower plot excited element upper plot adjacent element

\section{REFERENCES}

[1] A. Gachagan, et al IEEE Trans Ultrasonics, Ferroelectrics and Frequency Control, Vol 43, No, 4, pp678-689, (1996)

[2] D.W. Schindel, et al IEEE Trans Ultrasonics, Ferroelectrics and Frequency Control Vol 42, No 1, pp42-50 (1995)

[3] M.I. Haller, \& B.T. Khuri-Yakub, IEEE Transaction on UFFC, 1996. 43(1): p. 1-6

[4] K.S. Ho, R.J. Bradley, D.R. Billson and D.A. Hutchins, Ultrasonics. 48, 1-5 (2008).

[5] A. Octavio et al Microsyst Technol Vol 17, pp1635-1644 (2011)

[6] Mackie, E et al Presented at IEEE IUS Dresden

[7] O'Leary, R.L. et al Proc IEEE IUS 2013, pp1113-16 doi 10.1109/ULTSYM.2013.0285

[8] R Pericet-Camara, ChemPhysChem Vol 9, pp 1738 - 1746 (2008)

[9] H. Ulmke et al, Chem. Eng. Technol. Vol 24, No 3, pp 265-268, (2001)

[10] G.L. Switzer Rev. Sci. Instrum. Vol. 62,pp 2765-2771 (1991);

[11] G. Müller \& D.W. Baudrand ISBN 9780852180389 